\title{
BLACK HOLES
}

\section{Some photons left behind}

Astrophys. J. 892, 47 (2020)

Within a black-hole binary (BHB), gas from the companion star feeds the accretion disk of a stellar-mass black hole. Gravity and friction heat up the inspiralling material in the disk, which emits X-ray photons. Riley Connors and co-workers discovered that photons escaping the galactic BHB XTE J1550-564 can get pulled backwards.

The authors modelled the data taken by the Rossi X-ray Timing Explorer satellite (retired in 2012) during two outbursts of XTE J1550-564 in 1998/99 and 2000 in order to understand the geometry and thermal properties of the inner disk and corona. Using the reflection model relxillNS, which treats the irradiating spectrum as a blackbody of a single (rather than multiple) temperature, they found that $\sim 5.2 \%$ of the soft photons return to the inner disk, in agreement with simulations of relativistic ray tracing $(\sim 5.4 \%)$. The returning photons then reflect off the disk and ultimately escape. Moreover, the best-fit disk inclinations are low (39\% compared to the binary inclination of $75 \%$; $90 \%$ would be edge-on, for instance), suggesting that the inner disk may be warped.

As variations in photon flux and in spectral properties for stellar BHBs occur on the scale of days to weeks, they can be used as analogues of active galactic nuclei, which do not vary on human timescales. Up next, the authors will try a fully relativistic, self-consistent model with a multi-temperature-disk blackbody spectrum.

May Chiao

Published online: 1 May 2020

https://doi.org/10.1038/s41550-020-1111-6 